\title{
Leucémies aiguës à chromosome Philadelphie : un nouveau bcr et un modèle de recombinaison
}

\author{
Sai Juan Chen, Zhu Chen, Christian-Jacques Larsen, Roland Berger
}

Le chromosome Philadelphie (Phl) a longtemps été considéré comme l'anomalie chromosomique spécifique d'une leucémie de l'adulte, la leucémie myéloïde chronique (LMC). Il résulte habituellement d'une translocation $\mathrm{t}(9 ; 22)$ avec des points de cassure stéréotypés sur les bandes chromosomiques 9q34 et $22 q 11$, et correspond donc à un échange de matériel chromosomique entre les chromosomes 9 et 22 . Il a été montré ensuite que le $\mathrm{Phl}$ pouvait se rencontrer également dans des leucémies aiguës, lymphoblastiques (LAL) surtout, mais aussi myéloblastiques (LAM). Les conséquences moléculaires de la translocation ont été largement étudiées ces dernières années. Le proto-oncogène $c$-abl, initialement découvert sous la forme activée d'un oncogène dans un rétrovirus murin, et normalement localisé sur la bande 9q34, est transféré sur le chromosome 22 remanié du fait de la translocation. L'analyse d'un grand nombre de LMC a montré qu'il existe une grande hétérogénéité des points de cassure dans le gène $c$-abl. En revanche, sur la bande $22 q 11$, les points de cassure de la LMC s'accumulent dans une région relativement limitée de 5,8 kb qui a été appelée pour cette raison bcr (pour breakpoint cluster region) [1]. L'analyse fine des remaniements a été faite à partir de sondes moléculaires : bcr constitue la partie médiane d'un gène de grande taille nommé $B C R$ (ce qui crée une confusion malheureuse). La translocation chromosomique, en juxtaposant une portion

\section{ADRESSE}

S.J. Chen: docteur en médecine, docteur ès sciences. Z. Chen : docteur en médecine, docteur ès sciences. C.-J. Larsen: directeur de recherce au Cnrs. R. Berger: directeur de recherche au Cnrs. Inserm U. 301, hôpital Saint-Louis, l, avenue Claude-Vellefaux 75475 Paris Cedex 10, France.

TIRÉS A PART

\section{R. Berger.}

proximale du gène $B C R$ à la partie distale du proto-oncogène $c$-abl, engendre un nouveau gène chimérique qui est transcrit dans les cellules leucémiques sous la forme d'un ARN messager de 8,5 kb (au lieu de $6,5-7 \mathrm{~kb}$ pour l'ARNm du gène $c$ $a b l)$. En dépit du remaniement, cet ARN a gardé une capacité codante et est lui-même traduit. Les masses moléculaires des protéines $\mathrm{Bcr}$-Abl et Abl diffèrent sensiblement (respectivement 210 et $145 \mathrm{kDa}$ ) et la protéine chimérique a une activité tyrosine kinase prononcée [2].

Dans les LAL à Phl, la protéine BcrAbl est de $210 \mathrm{kDa}$ comme dans la LMC dans la moitié des cas, mais dans l'autre moitié, une protéine de 190 kDa a été détectée, qui est traduite à partir d'un ARNm de $7 \mathrm{~kb}$ [3]. La question se posait donc de savoir où était situé le point de cassure dans BCR dans ces cas. Par ailleurs, l'existence de cette nouvelle catégorie de produit $\mathrm{Bcr}-\mathrm{Abl}$ ne reflétait-elle pas une différence entre LAL et LAM à $\mathrm{Phl}$ ?

Pour répondre à ces questions, nous avons étudié huit LAL et six LAM à Phl [4]. Le point de cassure était localisé dans le segment bcr dans trois cas de LAM et dans trois cas de LAL. La structure du Phl était donc dans ces cas du même type que celle des LMC. En revanche, la détection d'un ARNm bcr-abl de $7 \mathrm{~kb}$ dans les huit autres cas suggérait que le point de cassure sur le chromosome 22 pouvait se localiser dans une région plus en amont du gène $B C R$, ainsi que l'étude de deux cas l'avait montré [5]. Nous avons décidé de «marcher " sur le chromosome 22 en remontant de 3' vers 5' afin de localiser les points de cassure. Nous avons pu montrer [6] que dans six cas sur huit les points de cassure se situaient dans un segment de $10,8 \mathrm{~kb}$ appartenant au premier intron du gène $B C R$. Plus précisément, ce segment est situé à environ $40 \mathrm{~kb}$ en 5 , de la région bcr. Cette accumulation dans une région relativement limitée d'un gène de grande taille suggérait l'existence d'une deuxième breakpoint cluster region que nous avons appelée provisoirement bcr2. Continuant à « marcher » sur le chromosome 22 , nous avons trouvé que les points de cassure des deux derniers cas de LA étaient localisés dans un segment de $5 \mathrm{~kb}$ situé $16 \mathrm{~kb}$ plus en 5' dans le premier intron de $B C R$ [7], suggérant qu'il pouvait exister une troisième région préférentielle des points de cassure («bcr3»?). A ce jour, il n'existe aucune corrélation entre les sites des points de cassure et le type de différenciation lymphoblastique ou myéloblastique de la leucémie aiguë.

Pour tenter de comprendre le mécanisme de la formation de la translocation à l'origine du $\mathrm{Phl}$ des LA, nous avons établi la séquence nucléotidique d'un point de cassure dans bcr 2 , après clonage des segments d'ADN entourant les points de cassure sur les chromosomes 9 et 22 remaniés [8]. Les séquences des régions correspondantes des chromosomes 9 et 22 normaux ont été également établies. La comparaison des séquences d'ADN des régions normales et remaniées a révélé une conservation quasi complète de la séquence normale puisque, dans le cas de ce patient, deux nucléotides seulement avaient été perdus du fait de la translocation et aucun n'avait été ajouté. Cette conservation par rapport aux régions homologues normales ainsi que l'absence de séquence signal de recombinaison de type heptamère-nonamère qui président aux recombinaisons somatiques des gènes d'immunoglobulines, permet d'éliminer un mécanisme de recombinaison illégitime similaire à celui des proliférations lymphoïdes malignes où l'on a incri-

* Famille de séquences répétées dans le génome humain, où il en existe près de 300000 copies par jeu de chromosomes. 
miné la recombinase reconnaissant ces séquences signal. En revanche, deux séquences Alu* (note p. 282), inversées ont été trouvées sur les chromosomes 9 et 22 normaux, séparées respectivement par environ 90 et 210 bases. En outre le point de cassure est situé dans l'une de ces séquences Alu appartenant à la même sous-famille sur les deux chromosomes. On peut donc proposer un modèle possible pour expliquer la formation de l'appariement illégitime entre les chromosomes 9 et 22 : l'appariement des deux séquences Alu crée une double structure en épingle à cheveux qui permet l'accès au complexe protéique responsable de la recombinaison anormale (figure 2). La translocation résulterait alors de la cassure et du recollement anormal des segments impliqués. Pour généraliser un tel modèle, il convient d'examiner d'autres translocations du même type, puis d'essayer d'identifier le complexe enzymatique

\section{RÉFÉRENCES}

1. Groffen J, Stephenson JR, Heisterkamp N, et al. Philadelphia chromosomal breakpoints are clustered within a limited region of bcr on chromosome 22. Cell $1984 ; 36: 93-9$.

2. Groffen J, Heisterkamp N, Stam K. Activation of $\mathrm{c}-\mathrm{abl}$ as a result of the $\mathrm{Ph}^{\prime}$ translocation in chronic myelocytic leukemia. $A d v$ Virol Oncol 1987; 7 : 77-97.

3. Kurzrock R, Gutterman JU, Talpaz M. The molecular genetics of Philadelphia chromosome-positive leukemias. N Engl J Med 1988 ; 319: 990-8.

4. Chen SJ, Flandrin G, Daniel MT, et al. Philadelphia-positive acute non lymphocytic leukemia : lineage promiscuity and inconsistently rearranged breakpoint cluster region (bcr). Leukemia 1988; 2 : 261-73.

5. Hermans A, Heisterkamp N, Von Lindern $\mathrm{M}$, et al. Unique fusion of bcr and c-abl genes in Philadelphia-positive acute lymphoblastic leukemia. Cell 1987; 51 : 33-40.

6. Chen S J, Chen Z, Grausz D, et al. Molecular cloning of a 5' segment of the genomic Phl gene defines a new breakpoint cluster region (bcr2) in Philadelphia-positive acute leukemias. Leukemia 1988; 2: 634-41.

7. Chen SJ, Chen Z, Hillion J, et al. Ph-1positive, bcr negative acute leukemias : clustering of breakpoints on chromosome 22 in the 3 ' end of BCR gene first intron. Blood (sous presse).

8. Chen S J, Chen Z, d'Auriol L, et al. $\mathrm{Phl}^{+} \mathrm{bcr}-$ acute leukemias: implication of Alu sequences in a chromosomal translocation occurring in the new cluster region within the BCR gene. Oncogene $1989 ; 4$ : 195202.

\section{Summary}

Philadelphia chromosome-positive acute leukemia : a new bcr and a model of recombination

A molecular study of 14 cases of $\mathrm{Phl}+\mathrm{ALL}$ and AML was performed with molecular probes covering the CML breakpoint cluster region (bcr) of the BCR gene, and the first intron $(70 \mathrm{~kb})$ of this gene. While six cases possessed a classical bcr rearrangement, in six other cases the breakpoints on chromosome 22 were clustered in a $10.8 \mathrm{~kb}$ DNA segment localized in the 3' end of the BCR intron 1. This new cluster of breakpoints located at $40 \mathrm{~kb}$ upstream the classical bcr was tentatively designated bcr2. Sequence determination of rearranged chromosomes 9 and 22 and their normal counterparts in one patient with a bcr2 rearrangement indicated the presence of repeated Alu sequences in the close vicinity of the breakpoints, suggesting the involvement of these sequences in the illegitimate recombination mechanism which generated the translocation.
Figure 1. Carte de restriction partielle du gène BCR. Les rectangles rouges indiquent les exons, et les segments bcr, bcr 2 et bcr3 sont détaillés (en bas). Les flèches noires indiquent les points de cassure de 14 cas de leucémie aiguë à chromosome Philadelphie (Ph1). E: EcoRI, B: BamHI, Bg : $B g / 1 I I, H$ : HindIII.

Figure 2. Mécanisme possible de la formation de la translocation $t$ (9; 22). 1 : Séquences Alu inversées sur les chromosomes 9 et 22 et structure en épingle à cheveux. Les séquences de la même sous-famille sont en noir ou hachurées. Le chromosome 22 est représenté en rouge en dehors des séquences Alu. 2: Double structure en épingle à cheveux résultant de l'appariement illégitime des séquences Alu des chromosomes 9 et 22. Les flèches courtes indiquent les points de cassure. 3: Résultat de la recombinaison illégitime montrant les chromosomes 9 et 22 remaniés.
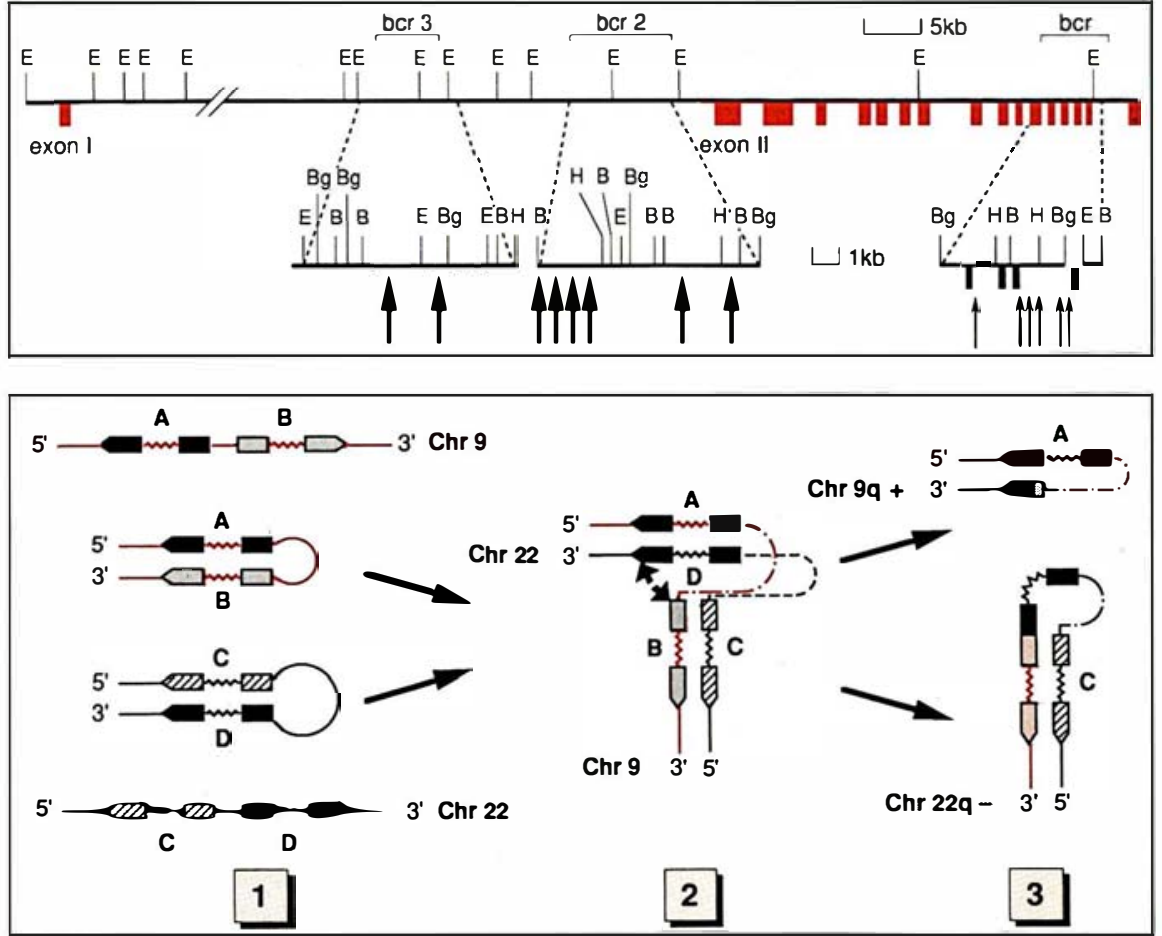